Revue d'histoire de l'Amérique française

ZRS REVUE D.HISTOIRE DE L'AMÉRIQUE FRANÇAISE

\title{
Les Documents de Lévis aux Archives canadiennes
}

\section{Lucien Brault}

Volume 4, numéro 4, mars 1951

URI : https://id.erudit.org/iderudit/801670ar

DOI : https://doi.org/10.7202/801670ar

Aller au sommaire du numéro

Éditeur(s)

Institut d'histoire de l'Amérique française

ISSN

0035-2357 (imprimé)

1492-1383 (numérique)

Découvrir la revue

Citer cet article

Brault, L. (1951). Les Documents de Lévis aux Archives canadiennes. Revue d'histoire de l'Amérique française, 4(4), 550-559.

https://doi.org/10.7202/801670ar d'utilisation que vous pouvez consulter en ligne.

https://apropos.erudit.org/fr/usagers/politique-dutilisation/ 


\section{LES DOCUMENTS DE LÉVIS}

\section{AUX ARCHIVES CANADIENNES}

Les Archives publiques du Canada ont fait l'acquisition, récemment, de neuf des onze volumes des documents du chevalier François-Gaston de Lévis. Cette série ajoute considérablement à la collection déjà fameuse de manuscrits originaux, se rapportant à la guerre de 1760 , possédée par les Archives canadiennes, soit les papiers de Monckton, ceux de Townshend et la collection Bourlamaque qui comprend des lettres de Vaudreuil, de Montcalm, etc.

Formée par le chevalier de Lévis après la mort de Montcalm et la chute de Québec, cette série de documents comprend la correspondance personnelle de ce soldat distingué avec les Grands du temps. Elle fut conservée dans les archives privées de la famille de Lévis jusqu'à ces dernières années.

En 1888, au cours d'un voyage de recherches a Paris, l'abbé Henri-Raymond Casgrain en prit connaissance, chez le Comte Raimond de Nicolay, neveu et héritier du dernier duc de Lévis (1863) et arrière petit-fils du chevalier. A la demande de l'abbé Casgrain, le comte de Nicolay permit au gouvernement de la province de Québec de publier ces documents en hommage au grand héros de la bataille de Sainte-Foy. C'est la série de douze volumes éditée par l'abbé Casgrain lui-même et intitulée "Collection des Manuscrits du maréchal de Lévis, Québec, 1889-1895", que tous les chercheurs et historiens canadiens connaissent.

Ici, pour l'historien se pose la question: y a-t-il variantes considérables entre les textes publiés par Casgrain et les textes nouvellement acquis par les archives? Si l'on compare l'original avec la publication de l'abbé Casgrain, on se rend compte que celle-ci n'est pas complète, ni totalement exacte. Ainsi, dans les volumes imprimés, on remarque des additions et des omissions, c'est-à-dire des lettres qui ne se trouvent pas dans l'original et des documents originaux et 
certains passages qui ne sont pas publiés. Des passages effacés, mais encore lisibles dans le manuscrit, ont été omis, comme à la page $133 \mathrm{du}$ volume I. Lorsque au volume II, Lévis raconte les mêmes événements à deux personnes différentes une seule lettre est imprimée. Cependant qu'il soit dit immédiatement que nous ne devons pas tenir l'abbé Casgrain responsable de ces inexactitudes, car nous ne possédons pas la copie qui a servi à l'impression et nous savons que L. Lecestre a fait la transcription pour le Comte de Nicolay. Il nous est donc impossible de savoir qui a fait les erreurs.

Voici une brève description de cette série avec les quelques différences déjà notées entre l'original et l'imprimé.

\section{Volume 1}

“Journal des campagnes du chevalier de Lévis en Canada de 1756 à 1760.

Ce volume de 285 pages est écrit de la main d'un secrétaire. Il comprend neuf grandes cartes géographiques contemporaines, sept manuscrites, dont six en couleurs, et deux imprimées. Les diverses batailles de la campagne de $1756-1760$ y sont indiquées en détails. Ces cartes n'ont pas été publiées par l'abbé Casgrain. Elles représentent:

1. Le champ de bataille et l'action au fort Duquesne, le 14 septembre 1758, alors que les Français, sous le commandement de Ligneris, remportèrent une victoire sur les armées anglaises commandées par le major Grant.

2. Une vue de la bataille du 8 septembre 1755, au lac SaintSacrement (George), ou Dieskau a subi une défaite aux mains de sir William Johnson.

3. Un plan des forts de Chouaguen, indiquant le débarquement, le camp et les attaques de l'armée commandée par Montcalm en août 1756 .

4. Un plan du fort William Henry ou fort George construit par les Anglais en 1756, assiégé et pris en aout 1757, par l'armée francaise commandée par Montcalm.

5. Un plan des retranchements sur les hauteurs en avant du 
fort de Carillon (Ticondéroga) attaqués par 25,000 Anglais, sous Abercromby, le 8 juillet 1758, et défendus par 3,600 Français commandés par Montcalm.

6. L'attaque et la prise du fort Niagara par les Anglais commandés par Johnson, le 25 juillet 1759.

7. Un plan exact des environs de Québec montrant les plaines d'Abraham où eut lieu la bataille du 13 septembre 1759.

8. Un plan de Québec et de la bataille qui s'est livrée dans ces environs le 28 avril 1760, entre les Français commandés par Lévis et les Anglais, par James Murray.

9. La prise du fort Lévis par les Anglais le 25 août 1760.

Ces cartes géographiques sont donc des additions à l'ouvrage imprimé.

\section{Volume 2}

Lettres du chevalier de Lévis concernant la guerre du Canada (17561760).

Ce volume contient 186 documents, probablement la copie des lettres et des mémoires envoyés par Lévis et transcrits par un secrétaire. Trente ont été adressées au marquis de Vaudreuil, quatre, au marquis de Montcalm, sept, au roi de Pologne, etc. Une lettre à Murray est remarquable par sa courtoisie. A cette époque, il semble qu'une fois le combat terminé, les sentiments redevenaient humains beaucoup plus rapidement que de nos jours. Ainsi le général anglais envoya du vin pour les malades et blessés français, et du fromage pour Lévis, et ce dernier, en retour, envoya des jeux et deux paquets de pruche, pour la fabrication d'un remède contre le scorbut.

\section{Volume 3}

Lettres de la cour de Versailles au baron de Dieskau, au marquis de Montcalm et au chevalier de Lévis.

Environ 145 lettres et documents, dont quelques-uns sont des duplicatas, d'autres, des copies. 
Ici, on trouve quelques divergences avec la version publiée. Par exemple, les documents imprimés suivants n'apparaissent pas dans le manuscrit:

No 34. Congé temporaire à un lieutenant du second bataillon du régiment d'infanterie de la Sarre.

Nos 42 et 43. Deux nominations de lieutenants réformés au second bataillon du régiment d'infanterie de la Sarre et au second bataillon du régiment d'infanterie de Guyenne.

Nos 46 et 47. Deux lettres chiffrées de Bougainville à Montcalm.

No 109. Une lettre de Louis XV nommant le sieur de Boischâtel, chevalier de Saint-Louis.

No 126. Une lettre du duc de Choiseul, 1761.

Par contre, les deux lettres suivantes se trouvent dans le manuscrit et non dans l'imprimé. L'une est de William Pitt, datée de Whitehall, le 24 mars 1761, au maréchal de Lévis, accordant à ce dernier la permission de servir en Europe; l'autre est du maréchal Ligonier. Elle est datée de Londres le 10 mars 1761. Ces deux documents sont des exemples de charmante courtoisie à la mode du XVIIIe siècle et qu'il fait bon lire. En voici le texte:

Monsieur

J ay Receu avec Plaisir La Lettre que vous mavés fait Lhonour de mecrire puisquelle me Rapele le Souvenir d'un Parent et d'un amy de Monsieur et de Madame de Mirepoix que j'honorois infiniment; Jay eu L'honeur de mettre sous les yeux de sa Majesté Le desir que vous avés de servir, et Les advantages qui vous en Resulteront, La maniere Genereuse avec Laquelle vous aves traite nos anglois, a dabord determiné Sa Majesté d'accorder vostre demande vous Etes done en Liberté Monsieur de servir en Europe seulement; sy Le Roy exepte L'amerique, cest vostre faute, vous I avés servi avec trop de distinction. Je souhaite monsieur tout ce qui peut contribuer a vostre satisfaction, puisque Jay L'honeur detre avec La Plus Parfaite consideration

Monsieur

a Londres le Vostre tres humble
et tres obeissant serviteur $10^{\mathrm{me}}$ de mars 1761 Le Marech $^{\text {le }}$ vicomte Ligonier. 
Monsieur,

à Whitehall ce 24..$^{\circ}$ Mars 1761.

Ce m'est un vrai plaisir de pouvoir vous apprendre l'agréable nouvelle, que le Roi m'a autorisé à vous dire, que malgré la Capitulation faite entre Mons. ${ }^{2}$ le General Amberst et Mons. ${ }^{x}$ de Vaudreuil, vous avez la liberté de servir, pouvê que ce soit en Europe. Je me flate, Monsieur, que cette restriction ne sauroit vous être trop génante, ni préjudiciable en aucune façon à vos vues d'avancement.

C'est avec bien du regret que j'ai été dans l'impossibilité de vous faire reponse plutôt, m'étant trouvé pendant quelques semaines alité par un severe accès de Goute; ainsi que de n'être pas apresent à même de me servir de ma Plume pour vous assurer des sentimens de l'estime sincere et de la considération la plus parfaite avec lesquels j'ai l'honneur d'être,

Monsieur,

Votre très humble \& très obéissant serviteur

M. le Chev. ${ }^{\mathbf{r}}$ de Levis.

W. Pitt.

\section{VOLUME 4}

Pièces militaires.

Ce volume, de même que le onzième, n'est pas disponible. Tous deux font partie des archives d'un collectionneur d'autographes, aux Etats-Unis.

\section{VOLUME 5}

Lettres de M. de Bourlamaque au chevalier de Lévis.

Ce volume couvre la période du 26 mai 1759 au 2 septembre 1760 et comprend 76 lettres, dont la plupart autographiées.

La version imprimée omet la lettre suivante du 22 aout 1760:

a Saint ours sur le fleuve le 22 a 7 h $1 / 2$ du matin.

J'apprends dans le moment, mon cher general, que Sorel est abandonné j'y avois laissé $M^{r}$ Bellot avec ordre de se 
replier s'il étoit attaqué par une force superieure et de prendre garde de n'être pas coupé. Cette nuit les Anglois ont laissé deriver une vingtaine de Batteaux audessous de Sorel vers les maisons du bas de Sorel et ont fait en même tems sur leur flote, c'est a dire sur la division qui y est, un grand bruit de rames et de gens qui s'embarquent. les vingt batteaux ont mis a terre au bas des maisons et n'y ayant personne ont mis le feu partout et se sont approchés ainsi Bruslant du petit bois qui les separoit de Sorel. $\mathbf{M}^{\mathrm{r}}$ Bellot y avoit un poste sur un pont: il a envoié ordre aux postes de se replier et s'est replié lui même jusqu'a une lieuë au dessus de l'église il y a traversé de ce costé cy et attendoit la des ordres. Je lui ai mandé de s'y maintenir le plus qu'il pourroit. Et j'ai fait filer jusqu'a lai $M^{\mathbf{r}}$ de renegnaut (?) avec $\mathbf{1 8 0}$ hommes. l'ennemy n'a point attaqué Sorel cette nuit, mais surement au point du jour voiant le poste abandonné de dessus ses vaisseaux et s'y sera etabli J'attens le mouvement des anglois pour me determiner a ce que je dois faire. Je ne doute pas qu'il ne remonte la rivière Sorel et qu'il ne pousse trés viste le corps qui y est. Le fond de ce corps seroit de cinquante hommes si l'on pouvoit compter sur les Canadiens mais tous ceux de Sorel ont déjà abandonné et les autres sont eparpillés dans le bois. on ne pourra compter que sur les 100 soldats qui font le fond de ce corps. Je suivrai exactement les ordres que vous m'avés donné et si l'escadre ou au moins les troupes de debarquement entrent dans la rivière Sorel et la remontent je me porterai a Vercheres.

J'ai l'honneur d'être avec respect Monsieur Vôtre três humble et tres obeissant serviteur.

Bourlamaque.

L'escadre n'a pas bougé depuis ma dernière lettre il est vrai que le cour (?) est contraire. une Barque a passé le lac pour aller a quebec. Montreal

A Monsieur Le $\mathrm{Ch}^{\mathrm{r}}$ de Levis Marechal de Camp A

Pour une raison impossible à expliquer, le volume imprimé contient des lettres de Montcalm à Bourlamaque, lettres qui, au moment de l'impression du volume, faisaient partie de la collection de sir Thomas Phillips, et qui n'ont jamais fait partie de la collection de Lévis. 
Volume 6

Lettre du marquis de Montcalm au chevalier de Lévis.

Soit 153 documents datés de 1756 à 1758, dont plusieurs autographiés. Outre certains passages de lettres, la copie suivante du manuscrit n'apparaît pas à l'imprimé:

Ce 5 (mars 1759?)

Je dois repondre mon cher chevalier a vos deux dernieres lettres dont l'une par beland (?), boishebert et beaubassin ne partiront inter nos que du 20 au 25 d'ailleurs je ne dois pas pousser la faiblesse trop loin ny avoir l'air d'etre dependant de mon gout, voila le tems de se rendre a Montreal je pars demain je pars racomodé et cherchant à croire que les circonstances ont plus de tort que la personne un rendes vous doné avant hier tandis que les deux incomodes dinoient à l'intendance a fait un epanchement de cour ou l'on a mis un air de vérité qui prouve que l'on veut me resister et qui je l'avoue ma fait plaisir et m'a ramené entierement et remis en terme il vaut encor mieux je crois en avoir agi ainsi brules ma lettre je pars demain. au reste en voila pour huit mois a moins que le service du roy ne me ramenat a quebec, et un tems ou il faut l'oublier quoiqu'aymable et la femme qui me plaix le plus et le plus capable de me faire faire une sotise croyez moy autant que je vous ayme.

A Monsieur le chevalier de levis brigadier des armees. A Montreal.

A celui qui veut lire entre les lignes, cette lettre est révélatrice du caractère, de la conduite et de la vie intime de Montcalm au Canada.

Dans ce volume on trouve, en date du 14 septembre 1759, une lettre de Marcel, le troisième aide-de-camp et secrétaire de Montcalm, annonçant la mort de ce dernier et la défaite des Plaines d'Abraham. Plus de soixante lettres sont olographes et beaucoup d'autres contiennent des passages autographes. Ce volume a une grande valeur archivistique, car les documents olographes de Montcalm sont plutôt rares. Par contre, ils sont difficiles à relire. 


\section{VOLUMe 7}

Le Journal du marquis de Montcalm durant ses campagnes en Canada de 1756 a 1759, 550 pages.

De retour en France, Lévis fit relier ce volume pour son usage personnel. Ce journal est divisé en huit parties.

1. - Du 31 janvier au mois de novembre 1756: La prise d'Oswego.

2. - Du 10 novembre 1756 au 3 juillet 1757: Expéditions contre les Anglais et conseils de guerre avec les sauvages.

3. - Du 12 juillet au 31 aout 1757: Prise du fort George (William Henry).

. 4. - Du 1er septembre 1757 au 31 mai 1758: Commencement des difficultés dans les approvisionnements.

5. - Du 1er juin au 12 aout 1758: Refoulement des Anglais à Carillon (Ticondéroga).

6. - Du 13 aout 1758 au 27 mars 1759: Chute du fort Frontenac; difficultés grandissantes dans les approvisionnements.

7. - Du 3 avril au 14 mai 1759: Difficultés encore plus sérieuses; le renfort français n'arrive pas.

8. - Du 23 mai au 24 septembre 1759: Chute de Québec et mort de Montcalm.

Les sept premières parties sont de la main de Marcel et c'est, sans doute, Montcalm qui lui dicta les deux premières. Il semble que la troisième soit de F.-A. de Bougainville, premier aide-de-camp de Montcalm à ce moment. Les divisions quatre à sept sont également dictées par Montcalm ou écrites sous sa surveillance; on y rencontre, a maints endroits des mots et des passages entiers écrits de sa main. La huitième partie est d'une calligraphie différente et aurait peut-être été dictée par Marcel.

\section{Volume 8}

Lettres du marquis de Vaudreuil au chevalier de Lévis.

Soit environ 120 lettres datées du 27 mai 1756 au 2 septembre 1760, et 7 autres documents. Quelques lettres sont olographes. Il y 
en a également deux de Vaudreuil à Montcalm. Presque toutes les lettres portent la signature originale. La version imprimée comprend une lettre du 26 juillet 1756 qui n'apparaît pas au manuscrit et, par contre, l'extrait suivant de la lettre de Vaudreuil en date du 14 août 1756, est omis:

Grace a Dieu du succès que nous venons d'avoir a Choueguen. Je vous prie de ? faire rendre dans votre Camp. M. Laumonier ne fera pas de difficulté de chanter le te deum je luy garentis l'agreement de Mgr l'Eveque. vous fairez tirer du Canon, et une descharge de toute votre mousqueterie suivant l'usage, Sans cependant occasionner une trop grande consomation de poudre.

Vaudreuil

\section{Volume 9}

Lettres de l'intendant Bigot au chevalier de Lévis.

Soit 87 documents, couvrant la période du 20 juillet 1756 au 28 septembre 1760 .

\section{VOLUMe 10}

Lettres de divers particuliers au chevalier de Lévis.

Sur un total de 112 lettres, on en compte vingt-cinq, de Péan, 1756-1758; seize de Bernier, le commissaire de la guerre, 1759; neuf, de Bougainville, 1760; neuf, de Roquemaure, 1760; neuf, de Malartic, 1760; huit, du chevalier le Mercier, 1758-1759; huit, de Beauclair, 1759; sept, de Rigaud, le frère du gouverneur, 1759; et autres. Les deux lettres suivantes n'ont pas été publiées:

Sir./

I have the Honor of Your Letter of the 29th Ult. ${ }^{\circ}$; but as the Exchanging or Ransoming of prisoners, belonging to the Troops, is quite out of my province, I must referr You to General Amherst, or to the General here, for the settling that matter: I have, since the 18th instant, order'd all the prisoners taken by me, upon the River, before that Time, to be put on shore; the Number of which, infinitely 
exceds the Number of English Seamen taken since my arrival here.

When the Vessels come down the River, which I have permitted so to do, they shall be permitted to return with such Baggage of Your Officers as may have been left at Quebec.

I am, with very great Regard

Sterling Castle

1st October 1759

$\mathbf{S}^{\mathbf{r}}$

Your most obedient humble servant

Cha. ${ }^{s}$ Saunders.

Monsieur

A Quebec Le 14. ${ }^{\text {e }}$ Oct ${ }^{\text {bre }} 1759$

Monseigneur Le Gouverneur ayant Commandé que le Capitaine de Milices luy envoyat immédiatement un recensement exact des habitans de Votre Paroisse, Specifiant par leur noms les Peres, Meres, Garçons \& filles, aussy bien qu'une supputation de la quantité de Grains et un denombrement fidele des Bestiaux; Il exige que vous teniez la Main a ce que ses ordres sur ces differens articles soient duement executés. avec toute la Diligence Possible, et que vous Certifiez les etats en etre justes, Je suis

$$
\begin{aligned}
& \text { Monsieur } \\
& \text { Votre très obeissant serviteur } \\
& \text { H. T. Cramahé Sec, }{ }^{\text {re }}
\end{aligned}
$$

Volume 11

Ce volume, ainsi que le quatrième, n'est pas disponible étant aux mains d'un collectionneur d'autographes, aux États-Unis. Voilà, croyons-nous, pour aguicher la curiosité des chercheurs.

Lucien BraUlT, Directeur, Service des Recherches, Archives Publiques du Canada, Ottawa. 